\title{
Magnetic Resonance Imaging Features of Tumefactive Perivascular Spaces
}

\author{
Nail Bulakbașı (i) \\ Department of Radiology, University of Kyrenia School of Medicine, Kyrenia, Cyprus
}

ORCID iD of the author: N.B. 0000-000I-7528-8I07.

Cite this article as: Bulakbașı N. Magnetic Resonance Imaging Features of Tumefactive Perivascular Spaces. Cyprus J Med Sci 2020; 5(2): 167-70.

\begin{abstract}
The perivascular spaces (PVSs) are pia-lined potential spaces filled with interstitial fluid (ISF) and form a network throughout the brain, which is a part of glymphatic system and helps to derange of metabolites from cerebral parenchyma. The enlarged PVSs are the most common cystic lesion of the brain and gradually become prominent with age. Literally, tumefactive PVS is the giant enlargement of PVS, measuring $15 \mathrm{~mm}$ and more in size, and thought to be result of blocking of the outlet of ISF for any reason. Typically, they tend to from clusters of cysts, but tumefactive forms are usually solitary. They usually have moderate mass effect and can cause obstructive hydrocephalus when located in mesencephalothalamic region. Although they don't contain cerebrospinal fluid (CSF), they have similar signal to CSF on all magnetic resonance imaging (MRI) sequences and do not enhance. These MRI features are very useful for their differential diagnosis from other congenital, infectious, ischemic and tumoral cystic/necrotic lesions. The precise diagnosis of PVS is critical to prevent patients with "leave-me-alone" lesions, from unnecessary surgical interventions.
\end{abstract}

Keywords: Perivascular Spaces, Virchow-Robin Spaces, Glymphatic System, Magnetic Resonance Imaging

\section{INTRODUCTION}

From the historical perspective, the perivascular spaces (PVSs) were first described by the German pathologist Rudolf Virchow and French anatomist Charles Philippe Robin, so they were also called as Virchow-Robin spaces (I, 2). Until to the advent of magnetic resonance imaging (MRI) to neuroradiology, they have been reported as incidental findings at autopsies and classified as normal anatomical variant and/or result of aging brain, in literature (3-5). Following the widespread usage of MRI, they became more visible, because they were usually invisible on computed tomography (CT) images (6). They were also considered as normal variant in radiology literature $(3,4,6-8)$. Following the recognition of cerebral glymphatic system and the excretory role of PVSs in this system, they were started to be evaluated in more detail (9-II).

Under normal conditions, the PVSs are not recognizable on MRI in pediatric and adult population but become prominent with age and sometimes formed a cyst in the brain parenchyma. Enlarged PVSs are the most common parenchymal cysts in the human brain and usually considered as a normal variant. By general acceptance, they are "leave me alone" lesions that should not be mistaken for serious disease (6). Besides age, recent studies have also linked enlarged PVSs with lacunar stroke subtype and enlarged PVSs were considered as a potential biomarker of neurovascular and neurodegenerative diseases $(3,4,12-16)$.

\section{Histopathology}

The PVSs are pia-lined potential spaces filled with interstitial fluid (ISF), not cerebrospinal fluid (CSF) and formed by the invagination of the pia mater into the brain parenchyma along the penetrating cerebral arteries and draining veins (3, 7, 12). PVSs form a complex intraparenchymal network distributed over the whole brain, connecting the cerebral convexities, basal cisterns, and ventricular system but, the PVSs do not communicate directly with the subarachnoid space or CSF. PVSs network plays an important role both in providing drainage routes for cerebral metabolites and in maintaining normal intracranial pressure $(6,9-11)$. They are also part of the cerebral glymphatic system, in which CSF-ISF exchange occurs within brain parenchyma probably mediated by aquaporin 4 water channels and a substantial amount of ISF and cerebral metabolites, such as amyloid beta, exits the brain via connections between the PVSs and leptomeningeal vessels (9). By this way, the intravenous gadolinium-based contrast agents can also enter the PVSs (I0, II, I7). 

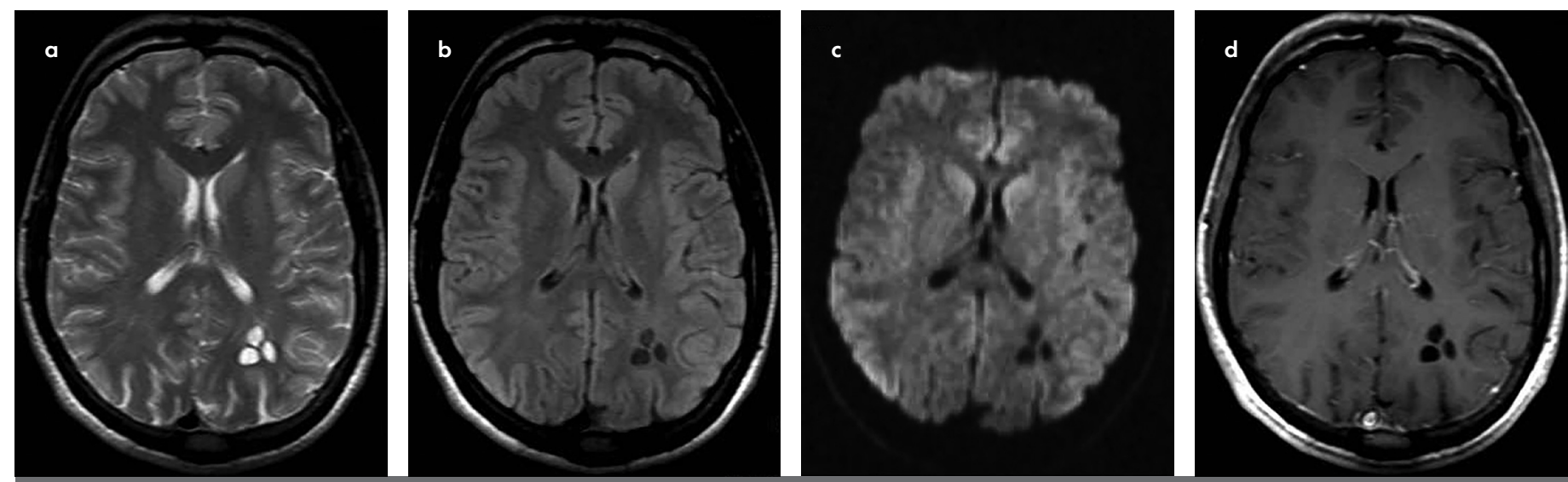

FIGURE I. a-d. Type I PVSs. Axial T2 (a), FLAIR (b), trace DWI (c) and postcontrast TI (d) images reveal the cluster of cysts having similar signal to CSF on all sequences without any prominent enhancement. They are asymmetrically located and follow the vascular distribution at the deep white matter of left occipital lobe.

They can be seen throughout brain wherever vessels are present, but mostly seen in the inferior third of the basal ganglia (clustered around the anterior commissure), subcortical white matter (including the external capsule), ponto-mesencephalic junction, midbrain and dentate nuclei of the cerebellum $(3,6-8)$. The PVSs are classified into three groups according to their location: type I (along the lenticulostriate arteries entering the basal ganglia through the anterior perforated substance), type 2 (along the perforating medullary arteries entering over high convexities through cortical gray matter), type 3 (along the penetrating collicular arteries in the mesencephalothalamic region) (8). Cortical PVSs (type 2) are lined by a single layer of pia, whereas two layers accompany lenticulostriate (type I) and collicular arteries (type 3) (5).

Type I and 2 tumefactive PVSs are usually asymptomatic or with nonspecific symptoms such as headache, dizziness, migraine, memory impairment, and Parkinson-like symptoms have been reported in some cases, but their relationship to enlarged PVSs is unclear (I316). About $75 \%$ of type 3 giant or tumefactive PVSs are symptomatic and can cause obstruction on CSF pathways, which occurs slowly over a long period of time, and can lead to noncommunicating type hydrocephalus (6-8) The patients with osbtructive hydrocephalus, can be treated either by ventriculoperitoneal shunting or endoscopic third ventriculostomy, or by cystoperitoneal shunting (7).

\section{Imaging Findings}

MRI is the modality of choice in the imaging of PVS and their detectability increases with higher MR strengths. Compare to I.5 T and less MR imagers, even very small PVSs can easily be detected by high-resolution 3T MRI in nearly all patients, in virtually every location, and at all ages. Additionally, PVSs can also be identified

\section{Main Points:}

- Tumefactive perivascular spaces are benign non-pathological conditions of cerebral white matter.

- Radiologically they mimic many pathological conditions including ischemic, congenital, infectious or tumoral cerebral cystic lesions.

- Unique MRI features of tumefactive PVSs having similar signal intensity to CSF on all MRI sequences and following the vascular distribution allow their differential diagnosis. in pediatric population on high-resolution MR scans. But, PVSs are not visible as they pass through cortex, even at 7T MRI (6).

PVSs are usually detected incidentally in routine MR images. The high-resolution volumetric fluid attenuated inversion recovery (FLAIR) or T2 weighted images have the highest diagnostic accuracy in the identification of PVSs. The postcontrast TI weighted images and other advanced MR imaging techniques such as; diffusion $(\mathrm{DWI})$, perfusion $(\mathrm{PWI})$, susceptibility $(\mathrm{SWI})$ weighted images are usually reserved for the differential diagnosis of PVSs from other congenital, infectious, ischemic and tumoral cystic/necrotic lesions.

In MRI, enlarged PVSs may be round, ovoid, linear or tubular in shape but always follow the vascular distribution (Figure I). This is one of the most important differential clues for their diagnosis. They may vary from solitary, unilocular, small, and unobtrusive lesions to multiple, large, bizarre, multilocular CSF-like fluid clusters, which leads to misdiagnosis of multicystic brain tumors. They have smooth and regular contours. Most of them are less than $15 \mathrm{~mm}$ in size $(5-8,18,19)$. Asymmetric distribution is common. Commonly, they can cause mass effect, and symptoms especially when located in the brainstem. Even though they are filled with ISF, PVSs usually follow CSF on all MRI sequences but, quantitative studies revealed statistically significant difference between PVSs and CSF, which is generally unrecognizable by human eye (20). This is the result of different contents of both spaces and supports the theory that PVSs are pia-lined potential spaces filled with interstitial fluid (ISF), not cerebrospinal fluid (CSF) (3, 5, 6). They always have high apparent diffusion coefficient (ADC) values like CSF on trace DWI images, which helps to differentiate them from the ischemic lesions having restricted diffusion (19). They don't have any calcification, hemorrhage or high protein content unlike other congenital, infectious or tumoral cerebral cystic lesions. About 75\% suppress completely on FLAIR images and $25 \%$ have hyperintense rim and $5-10 \%$ have hyperintensity in perilesional white matter (6). Typically, they do not enhance with intravenous gadolinium-based contrast agents.

Extensive enlargement of PVSs is a rare but important matter and is also referred as giant so-called tumefactive PVSs (Figure 2) $(4,7,18)$. These are usually bigger than $1.5 \mathrm{~cm}$, measuring up to $9 \mathrm{~cm}$ in diameter have been reported $(4,6,7,18)$. The precise cause of this cystic enlargement is still unknown. Most investigators believe 

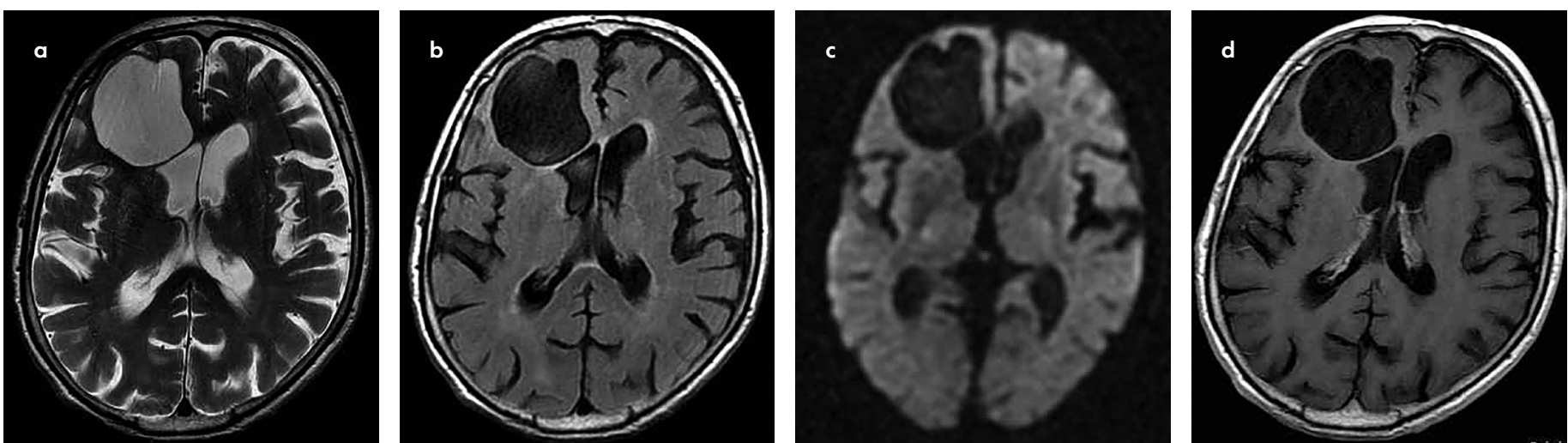

FIGURE 2. a-d. Tumefactive PVS. Axial T2 (a), FLAIR (b), trace DWI (c) and postcontrast TI (d) images reveal right frontal tumefactive PVS, having $45 \mathrm{~mm}$ highest diameter and similar signal to CSF on all sequences without any prominent enhancement. It has moderate mass effect to the right frontal ventricular horn.
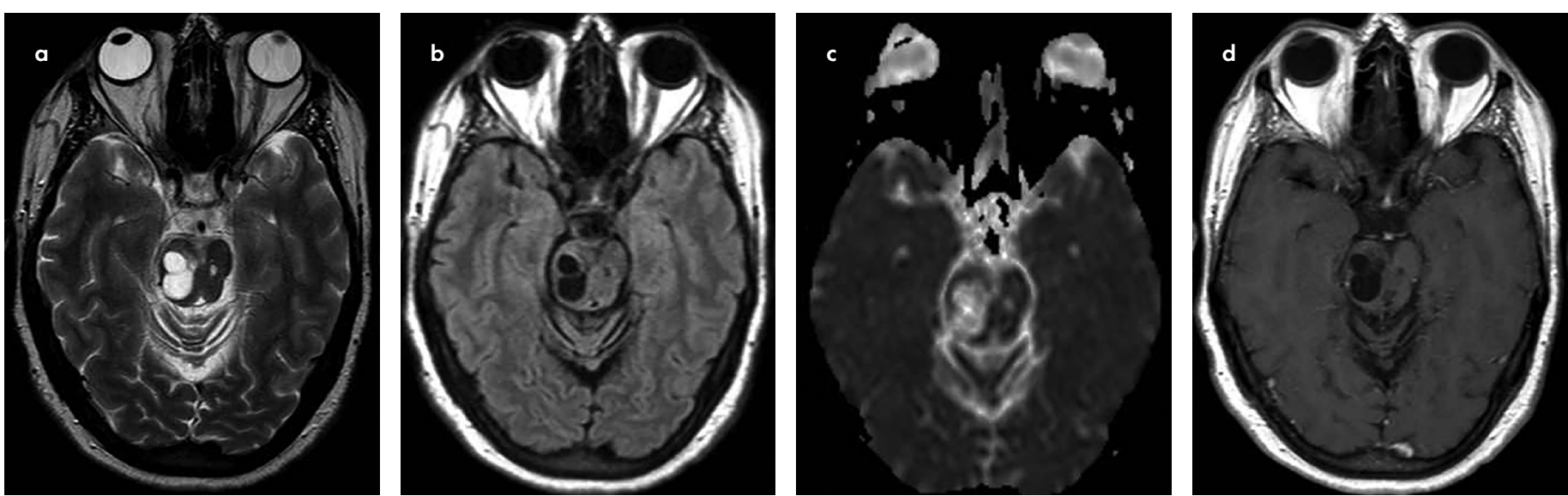

FIGURE 3. a-d. Tumefactive PVS. Axial T2 (a), FLAIR (b), trace ADC (c) and postcontrast TI (d) images reveal multiloculated tumefactive PVS at the right ponto-mesencephalic junction, having $20 \mathrm{~mm}$ highest diameter and similar signal to CSF on all sequences without any prominent enhancement. It has moderate mass effect to the brainstem and Sylvian aqueduct but not cause obstructive hydrocephalus yet.
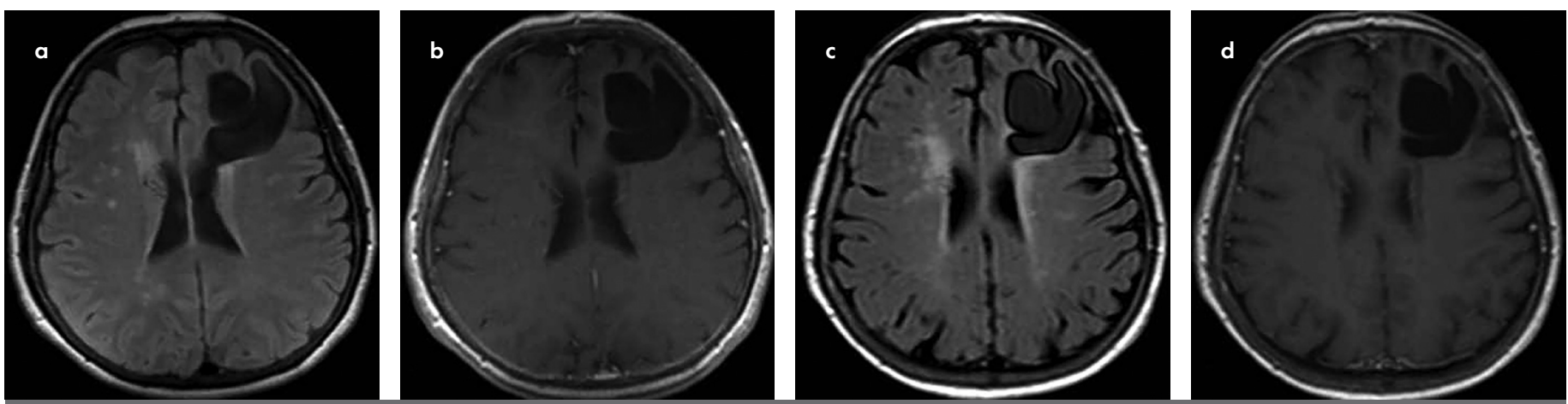

FIGURE 4. a-d. Tumefactive PVS. Axial FLAIR (a), and postcontrast TI (b) images reveal left multiloculated frontal tumefactive PVS, having similar signal to CSF on all sequences without any prominent enhancement but perilesional high signal on FLAIR (a) images resembling gliosis. On follow-up axial FLAIR (c), and postcontrast TI (d) images of the same patient obtained 2 years later, the size, appearance and signal characteristics of the PVS shows no change except mild increase in perilesional high signal on FLAIR (c)

that cystic enlargement of the PVSs is a result of blocking of the outlet of ISF for any reason, such as mechanical trauma due to CSF pulsation or vascular ectasia, increased vascular permeability resulting in fluid exudation into ISF or ischemic injury to perivascular tissue causing a secondary ex vacuo effect $(3,5,18)$. Similar to distribution of simple PVSs, tumefactive PVSs are also seen throughout the all cerebral vascular territories, but mostly seen in the mesencephalothalamic region (Figure 3) and frontal lobe (Figure 2) (7). They have also similar MRI features and one third of tumefactive
PVSs have surrounding T2/FLAIR hyperintensity, reflecting gliosis or edema $(3,5,18)$. When lesion becomes bigger (more than 2 $\mathrm{cm}$ ), the diagnosis becomes difficult because linear sign is lost and detection of relationship with the vascular distribution becomes harder (2I). At this point, detection of similar signal intensity and diffusion characteristic to CSF in all sequences, and the absence of contrast enhancement even in delayed images are very useful for their differential diagnosis from other tumor like lesions (17). The stability in size and appearance of tumefactive PVSs over time is 
critical for their diagnosis (Figure 4) although a few cases of progressively enlarging $\mathrm{PVS}$ s have been reported but the progression rate was not as fast as tumors had $(4,7,8,16)$.

\section{Differential Diagnosis}

The major differential diagnosis is chronic lacunar infarction. Although they often affect the basal ganglia and suppress on FLAIR, lacunar infarcts do not cluster around the anterior commissure, are often irregular in shape, and frequently exhibit hyperintensity in the adjacent brain. In acute stage, infarcts have low ADC values (diffusion restriction) on DWI and relative cerebral blood volume ( $\mathrm{CCBV}$ ) values on $\mathrm{PWI}$, but $\mathrm{ADC}$ values gradually increase with chronicity of the lesion, while rCB $\vee$ values remain low.

Another mimic is the neuroglial cysts, which are fluid-containing cavity buried within the cerebral white matter like enlarged PVSs. The neuroglial cysts are always solitary and located anywhere in the brain but not follow the vascular distribution. They have a similar appearance with enlarged PVSs on MRI but enlarged PVSs are usually multiple and form clusters.

The an intraparenchymal arachnoid cyst is quite rare compare to usual extra-axial location. They have also signal like CSF. It is often difficult to differentiate them from PVSs and neuroglial cysts on solely MRI findings. Contrary to arachnoid cysts, MRI demonstration of the communication of the cyst with the ventricle helps to differential diagnosis of the porencephalic cysts from other mimics.

Infectious cysts are usually small and represents with diffusion restriction and moderate to strong but irregular enhancing rim. In immunocompromised patients, enhancement of maybe faint or incomplete. Although often multiple or multilocular, they typically do not occur in clusters of variably sized cysts as is typical for enlarged PVSs. Diffusion restriction can be identified in the center of the infectious cyst, like in bacterial abscess due to presence of pus, or in the wall, like in tuberculous abscess due to inflammation.

Primary or metastatic cystic tumors may also resemble tumefactive PVSs, but even low-grade, tumors are usually enhanced, and have high perfusion and low diffusion values unlike PVSs. High rCBV values of primary or metastatic tumors on PWI help to differentiate them from other mimics.

\section{CONCLUSION}

Tumefactive PVSs are rare but important lesions, which must be differentiated from other ischemic, congenital, infectious or tumoral cerebral cystic lesions. The precise diagnosis of PVSs prevents patients from unnecessary surgical interventions. They have unique MRI features such as; they always follow the similar signal to CSF and the vascular distribution, usually they do not enhance, and sometimes have surrounding high T2/FLAIR signal due to edema or gliosis. The MRI features such as enhancement, diffusion and perfusion characteristics of other cystic lesions allow proper differential diagnosis of tumefactive PVSs, even they have reached to huge dimensions.

Peer-review: Externally peer-reviewed.

Conflict of Interest: Authors have no conflicts of interest to declare.
Financial Disclosure: The authors declared that this study has received no financial support.

\section{REFERENCES}

I. Virchow R, Ueber die erweiterung kleinerer gefaesse. Arch Pathol Anat Physiol Klin Med I851; 3: 427-62. [Crossref]

2. Robin C. Recherches sur quelques particularites de la structure des capillaires de l'encephale. J Physiol Homme Animaux 1859; 2: 537-48.

3. Adachi M, Hosoya T, Haku T, Yamaguchi K. Dilated Virchow-Robin spaces: MRI pathological study. Neuroradiology 1998; 40(I): 27-31. [Crossref]

4. Rudie JD, Rauschecker AM, Nabavizadeh SA, Mohan S. Neuroimaging of dilated perivascular spaces: From benign and pathologic causes to mimics. J Neuroimaging. 2018; 28(2): 139-49. [Crossref]

5. Zhang $E T$, Inman CB, Weller RO. Interrelationships of the pia mater and the perivascular (Virchow-Robin) spaces in the human cerebrum. J Anat 1990; 170: III-23.

6. Osborn AG. Chapter 28: Nonneoplastic cysts. In: Osborn AG, Hedlund GL, Salzman KL, editors. Osborn's Brain: Imaging, Pathology, And Anatomy. Second edition. Philadelphia: Elsevier; 2018.p.867-90I.

7. Kwee RM, Kwee TC: Tumefactive Virchow-Robin spaces. Eur J Radiol 2019; III: 21-33. [Crossref]

8. Kwee RM, Kwee TC. Virchow-Robin spaces at MR imaging. Radiographics. 2007; 27(4): I07I-86. [Crossref]

9. Rasmussen MK, Mestre H, Nedergaard M. The glymphatic pathway in neurological disorders. Lancet Neurol 2018; 17(II): I016-24. [Crossref]

10. Deike-Hofmann K, Reuter J, Haase R, Paech D, Gnirs R, Bickelhaupt $\mathrm{S}$, et al. Glymphatic pathway of gadolinium-based contrast agents through the brain: Overlooked and misinterpreted. Invest Radiol 2019; 54(4): 229-237. [Crossref]

II. Eide PK, Ringstad G. MRI with intrathecal MRI gadolinium contrast medium administration: a possible method to assess glymphatic function in human brain. Acta Radiol Open 2015; 4(II): 2058460115609635. [Crossref]

12. Pollock $\mathrm{H}$, Hutchings $\mathrm{M}$, Weller RO, Zhang ET. Perivascular spaces in the basal ganglia of the human brain: their relationship to lacunes. J Anat 1997; 19I(P+3): 337-46. [Crossref]

13. Bakker EN, Bacskai BJ, Arbel-Ornath M, Aldea R, Bedussi B, Morris $A W$, et al. Lymphatic clearance of the brain: perivascular, paravascular and significance for neurodegenerative diseases. Cell Mol Neurobiol 2016; 36(2): 181-94. [Crossref]

14. Ramirez J, Berezuk C, McNeely AA, Gao F, McLaurin J, Black SE. Imaging the perivascular space as a potential biomarker of neurovascular and neurodegenerative diseases. Cell Mol Neurobiol 20l6; 36(2): 289-99. [Crossref]

15. Zhang X, Ding L, Yang L, Qin W, Yuan J, Li S, et al. Brain atrophy correlates with severe enlarged perivascular spaces in basal ganglia among lacunar stroke patients PLoS One 20l6; II(2): e0I49593. [Crossref]

16. Machado MA Jr, Matos AS, Goyanna F, Barbosa VA, Vieira LC Dilatation of $V$ irchow-Robin spaces in patients with migraine. Arq Neuropsiquiatr 200I; 59(2-A): 206-9. [Crossref]

17. Naganawa S, Nakane T, Kawai H, Taoka T. Gd-based contrast enhancement of the perivascular spaces in the basal ganglia. Magn Reson Med Sci 2017; 16(I): 61-5. [Crossref]

18. Salzman KL, Osborn AG, House P, Jinkins JR, Ditchfield A, Cooper JA, et al. Giant tumefactive perivascular spaces. AJNR Am J Neuroradiol 2005; 26(2): 298-305

19. Yıldııım D, Gürses B, Kıbıcı K, Güvenç İ, Bulakbașı N, Tayfun C. MRI characterstics of atypically wide perivascular spaces. Yeditepe Medical Journal 2010: 14: 288-94. [Crossref]

20. Ozturk MH, Aydingoz U. Comparison of MR signal intensities of cerebral perivascular (Virchow-Robin) and subarachnoid spaces. J Comput Assist Tomogr 2002; 26: 902-4. [Crossref]

21. Sung J, Jang J, Choi HS, Jung SL, Ahn KJ, Kim BS. Linear sign in cystic brain lesions $\geq 5 \mathrm{~mm}$ : A suggestive feature of perivascular space. Eur Radiol 2017; 27(II): 4747-55. [Crossref] 